\title{
RESEARCH
}

Open Access

\section{The challenges of living with and managing epidermolysis bullosa: insights from patients and caregivers}

\author{
Anna L. Bruckner ${ }^{1 *}$, Michael Losow², Jayson Wisk², Nita Patel ${ }^{2}$, Allen Reha², Hjalmar Lagast ${ }^{2}$, Jamie Gault², \\ Jayne Gershkowitz ${ }^{2}$, Brett Kopelan ${ }^{3}$, Michael Hund ${ }^{4}$ and Dedee F. Murrell ${ }^{5}$
}

\begin{abstract}
Background: Little information is available regarding the burden of living with and managing epidermolysis bullosa, including the distinct challenges faced by patients with different disease types/subtypes.

Methods: A 90-question/item survey was developed to collect demographics, diagnostic data, management practices, and burden of illness information for patients with epidermolysis bullosa living in the United States. Recruitment was conducted via email and social media in partnership with epidermolysis bullosa patient advocacy organizations in the United States, and the survey was conducted via telephone interview by a third-party health research firm. Respondents aged $\geq 18$ years with a confirmed diagnosis of epidermolysis bullosa or caring for a patient with a confirmed diagnosis of epidermolysis bullosa were eligible to participate in the survey.

Results: In total, 156 responses were received from patients $(n=63)$ and caregivers $(n=93)$ representing the epidermolysis bullosa types of simplex, junctional, and dystrophic (subtypes: dominant and recessive). A large proportion of patients (21\%) and caregivers (32\%) reported that the condition was severe or very severe, and $19 \%$ of patients and $26 \%$ of caregivers reported a visit to an emergency department in the 12 months prior to the survey. Among the types/ subtypes represented, recessive dystrophic epidermolysis bullosa results in the greatest wound burden, with approximately $60 \%$ of patients and caregivers reporting wounds covering $>30 \%$ of total body area. Wound care is time consuming and commonly requires significant caregiver assistance. Therapeutic options are urgently needed and reducing the number and severity of wounds was generally ranked as the most important treatment factor.

Conclusions: Survey responses demonstrate that epidermolysis bullosa places a considerable burden on patients, their caregivers, and their families. The limitations caused by epidermolysis bullosa mean that both patients and caregivers must make difficult choices and compromises regarding education, career, and home life. Finally, survey results indicate that epidermolysis bullosa negatively impacts quality of life and causes financial burden to patients and their families.
\end{abstract}

Keywords: Caregivers, Disease burden, Epidermolysis bullosa, Management, Patients, Survey, Quality of life, Financial burden, Wound care

\footnotetext{
* Correspondence: anna.bruckner@cuanschutz.edu

${ }^{1}$ Children's Hospital Colorado, University of Colorado School of Medicine,

13123 E 16th Ave, B570, Aurora, CO 80045, USA

Full list of author information is available at the end of the article
}

(c) The Author(s). 2020 Open Access This article is distributed under the terms of the Creative Commons Attribution 4.0 International License (http://creativecommons.org/licenses/by/4.0/), which permits unrestricted use, distribution, and reproduction in any medium, provided you give appropriate credit to the original author(s) and the source, provide a link to the Creative Commons license, and indicate if changes were made. The Creative Commons Public Domain Dedication waiver (http://creativecommons.org/publicdomain/zero/1.0/) applies to the data made available in this article, unless otherwise stated. 


\section{Background}

Epidermolysis bullosa is a rare, often severe genetic disorder characterized by mechanical fragility and blistering or erosions of the skin, mucosa, or epithelial lining of organs in response to minimal trauma $[1,2]$. In addition to skin blistering, open wounds, and scarring, severe epidermolysis bullosa can produce extracutaneous manifestations including abnormalities of the gastrointestinal, cardiovascular, and genitourinary systems, as well as the eyes and oral cavities, and is associated with increased risk of premature death [2-4].

Multiple gene mutations affecting proteins responsible for skin integrity have been reported, including keratins (KRT5, KRT14), laminin-332 (LAMA3, LAMB3, LAMC2), dystonin epithelial isoform (DST), and collagen types VII and XVII (COL7A1, COL17A1) [4, 5]. These mutations result in several disease types and subtypes classified based upon the Stet of the cleavage plane in the skin, each with differing presentation $[3,4]$. The four major types of epidermolysis bullosa are epidermolysis bullosa simplex (70\%), dystrophic epidermolysis bullosa (25\%), junctional epidermolysis bullosa (5\%), and Kindler syndrome; however, the first 3 subtypes account for $\sim 99 \%$ of the patient population $[2,5,6]$.

The National Epidermolysis Bullosa Registry estimated that the overall prevalence of epidermolysis bullosa in the United States is 11.1 per one million live births, with an incidence of 1 in every 51,000 live births [7]. Others estimate the incidence of epidermolysis bullosa to be 1 in every 20,000 live births, with approximately 30,000 individuals affected in the United States [8]. Symptoms typically appear around the time of birth, although lesions may not appear in some individuals until adolescence or later, and accurate diagnosis may be delayed until adulthood [4].

Currently, there are no approved treatments for epidermolysis bullosa [9], and management focuses primarily on prevention of blistering, wound care, pain reduction, and early recognition and management of extracutaneous complications [1, 2, 10]. However, little information is available regarding patient and caregiver perspectives on the challenges of managing different types of epidermolysis bullosa and the burden of daily living with the condition [11].

We conducted a survey to understand the current manifestations and impact of epidermolysis bullosa from the patient or caregiver's perspective and to better understand the difficulties in dealing with various subtypes of the disease among patients residing in the United States.

\section{Methods}

\section{Survey design}

A 90-question/item survey was developed in partnership with epidermolysis bullosa patient advocacy organizations in the United States to collect demographics, diagnostic data, management practices, and burden of illness information on patients with epidermolysis bullosa (Additional file
$1)$. The survey was approved by a central institutional review board (New England Independent Review Board). The questions asked the patient or their caregiver to report on the patient, with the exception of one question that assessed life decisions made by the caregiver (for their own life) based on the patient's epidermolysis bullosa.

It was planned to recruit 200 patients into the survey. Recruitment was conducted via email and social media in partnership with epidermolysis bullosa patient advocacy organizations (Debra of America and EB Research Partnership) in the United States. The survey was conducted via telephone interview by a third-party health research firm (Engage Health, Eagan, MN). Recruitment and interviewing of patients and caregivers took place between April 11, 2017, and July 24, 2017.

Respondents were eligible to participate in the survey if they met the following criteria: aged $\geq 18$ years; confirmed diagnosis of epidermolysis bullosa or caring for a patient with a confirmed diagnosis of epidermolysis bullosa; and provided informed consent. Confirmed diagnosis of epidermolysis bullosa was established based on documentation from the patient's physician that listed both the name of the patient and the diagnosis of epidermolysis bullosa. Examples of these types of documents included, but were not limited to: physician encounter report/visit summary; physician note for special consideration (e.g., a note stating that a pediatric patient needed to bring scissors to camp for dressing changes); dressing change instructions; epidermolysis bullosa care plan; medical statement for receipt of social services; notification of genetic test results and diagnosis; copy of health record from patient portal; letter to referring physician from epidermolysis clinic; and copy of an email from healthcare provider to a patient confirming diagnosis.

\section{Analysis}

Descriptive statistics, including medians, ranges, and percentages, were used to report the results obtained from respondents. No statistical tests were performed.

\section{Results \\ Patients}

In total, 210 people accepted the initial invitation to participate in the survey. Of these, survey responses were received from 156 people in 39 states in the United States (Additional file 2). Fifty-four people, who had initially accepted the invitation to participate, were contacted but did not take part in the survey $(n=51$, never sent proof of an epidermolysis bullosa diagnosis; $n=3$, scheduled but did not call in for an interview). Individuals with a diagnosis of epidermolysis bullosa accounted for 63 responses (40.4\%), and the remaining 93 responses $(59.6 \%)$ came from caregivers.

Of the 63 patients who independently completed the survey, the median (range) age was 32 (18-70) years 
with more females $(n=47,74.6 \%)$ than males $(n=16$, $25.4 \%$ ). In contrast, the median (range) age of the patients whose caregivers provided responses was $7(0.2-$ 59) years with more males $(n=51,54.8 \%)$ than females $(n=42,45.2 \%)$. The composition of epidermolysis bullosa types were similar as reported by patients and caregivers; the overall composition was as follows: simplex ( $n=55,35.3 \%)$, junctional $(n=15,9.6 \%)$, dystrophic (subtypes: $n=31$, dominant [19.9\%] and $n=53$, recessive [34.0\%]), and unknown $(n=2,1.3 \%)$ (Table 1). For 119 patients, the first epidermolysis bullosa symptoms were noticed at birth. Of 150 respondents (patients and caregivers) who reported age at epidermolysis bullosa diagnosis, 56 (37.3\%) patients were diagnosed with epidermolysis bullosa at birth and 85 (56.7\%) more patients were diagnosed before the age of 1 year. $48.3 \%$ of patients (28 out of 58 ) and $40.7 \%$ of caregivers (37 out of 91) reported that first symptoms were noticed at birth; in addition, $34.5 \%$ of patients (20 out of 58 ) and $53.8 \%$ of caregivers (49 out of 91) reported that the time between first symptoms and diagnosis was 1 day to 1 year.

\section{Burden of disease}

Almost three-quarters of patients and caregivers rated the epidermolysis bullosa (their own or that of the patient) as moderate to very severe $(69.8 \%$ of patients $[n=$ 44] and $76.3 \%$ of caregivers $[n=71]$ ) (Fig. 1 ). On a scale of 1-5 (very mild-very severe), patients with recessive dystrophic epidermolysis bullosa had the highest mean rating of disease severity as reported by both patients and caregivers ( 3.2 by patients and 3.5 by caregivers), followed by patients with junctional epidermolysis bullosa ( 3.1 by both patients and caregivers), dominant dystrophic epidermolysis bullosa ( 3.1 by patients and 2.8 by caregivers), and epidermolysis bullosa simplex (2.4 by patients and 2.9 by caregivers).

Nearly all respondents $(95.2 \%$ of patients $[n=60]$ and 95.7\% of caregivers $[n=89]$ ) reported $\geq 1$ complication due to epidermolysis bullosa (Fig. 2). The median numbers of complications reported per individual by patient and caregiver were 7 and 6 , respectively. The maximum number of complications reported was 17 (reported by 1 patient and 1 caregiver). Nail abnormalities were common in all types of epidermolysis bullosa. Of note, patients with epidermolysis bullosa simplex had fewer oral cavity and dental problems than patients with other disease types. Conversely, more hand/foot contractures, anemia, esophageal strictures, and nutritional problems were reported in patients with recessive dystrophic epidermolysis bullosa compared with other disease types (Table 2).

In the 12 months prior to the survey, $19.0 \%$ of patients $(n=12)$ and $25.8 \%$ of caregivers $(n=24)$ had sought care for epidermolysis bullosa from an emergency department, with a mean number of 2 visits. Based on caregivers' response, patients with recessive dystrophic epidermolysis bullosa were more likely to visit an emergency department (14 out of 34 patients with this epidermolysis bullosa subtype, $41.2 \%)$ than patients with other disease subtypes (14.3 to 17.6\%). No apparent differences in emergency department visits between disease subtypes were observed based on patients' responses due to the small number of patient-reported visits to the emergency department.

\section{Wound burden}

About one-third of respondents $(31.7 \%$ of patients $[n=20]$ and $35.5 \%$ of caregivers $[n=33]$ ) reported that $>30 \%$ of the patient's total body area was covered by wounds; patients with recessive dystrophic epidermolysis bullosa were more likely to have $>30 \%$ of their body covered by wounds (57.9\% of patients and $61.8 \%$ of caregivers)(Table 3 ).

On a scale of 1 (none) to 10 (severe), patients rated their acute pain (mean score 4.7), chronic pain (4.4), and itch (5.7) in the last 2 weeks. Similarly, caregivers rated the patient's acute pain (mean score 5.7), chronic pain (3.8), and itch (5.4) in the last 2 weeks. Acute pain and itch were reported to be worse in patients with recessive dystrophic disease compared with other types/subtypes (Fig. 3).

Respondents reported that wound care can take several hours (Table 3), with $12.7 \%$ of patients and $9.7 \%$ of caregivers requiring $>4 \mathrm{~h}$ per day to care for wounds. The time required for wound care differed by epidermolysis bullosa type/subtype; patients with recessive dystrophic epidermolysis bullosa reported spending the longest amount of time on wound care whereas caregivers of patients with both junctional and recessive dystrophic subtypes reported spending the most time on wound care.

Patients and caregivers reported changing dressings on infected wounds more often than on noninfected wounds, and the frequency of wound dressing changes differed by epidermolysis bullosa type/subtype (Table 3 ). Overall, $74.6 \%$ of patients $(n=47)$ and $54.8 \%$ of caregivers $(n=51)$ stated that they changed the dressings on the same infected wound once or 2 to 3 times per day. In comparison, $42.9 \%$ of patients $(n=27)$ and $46.2 \%$ of caregivers $(n=43)$ changed the dressings on the same noninfected wound once or twice per day.

Most patients with epidermolysis bullosa required assistance with their wound care regimen. Caregivers reported that of the patients in their care, $66.7 \%$ always $(n=62), 18.3 \%$ sometimes $(n=17)$, and $15.1 \%$ never $(n=$ 14) required assistance. In contrast, among patients that independently participated in the survey, the frequency of assistance required was always by $12(19.0 \%)$ patients, sometimes by 19 (30.2\%) patients, and never by 32 $(50.8 \%)$ patients. This may partly reflect various patient factors, such as age (i.e., very young children would 


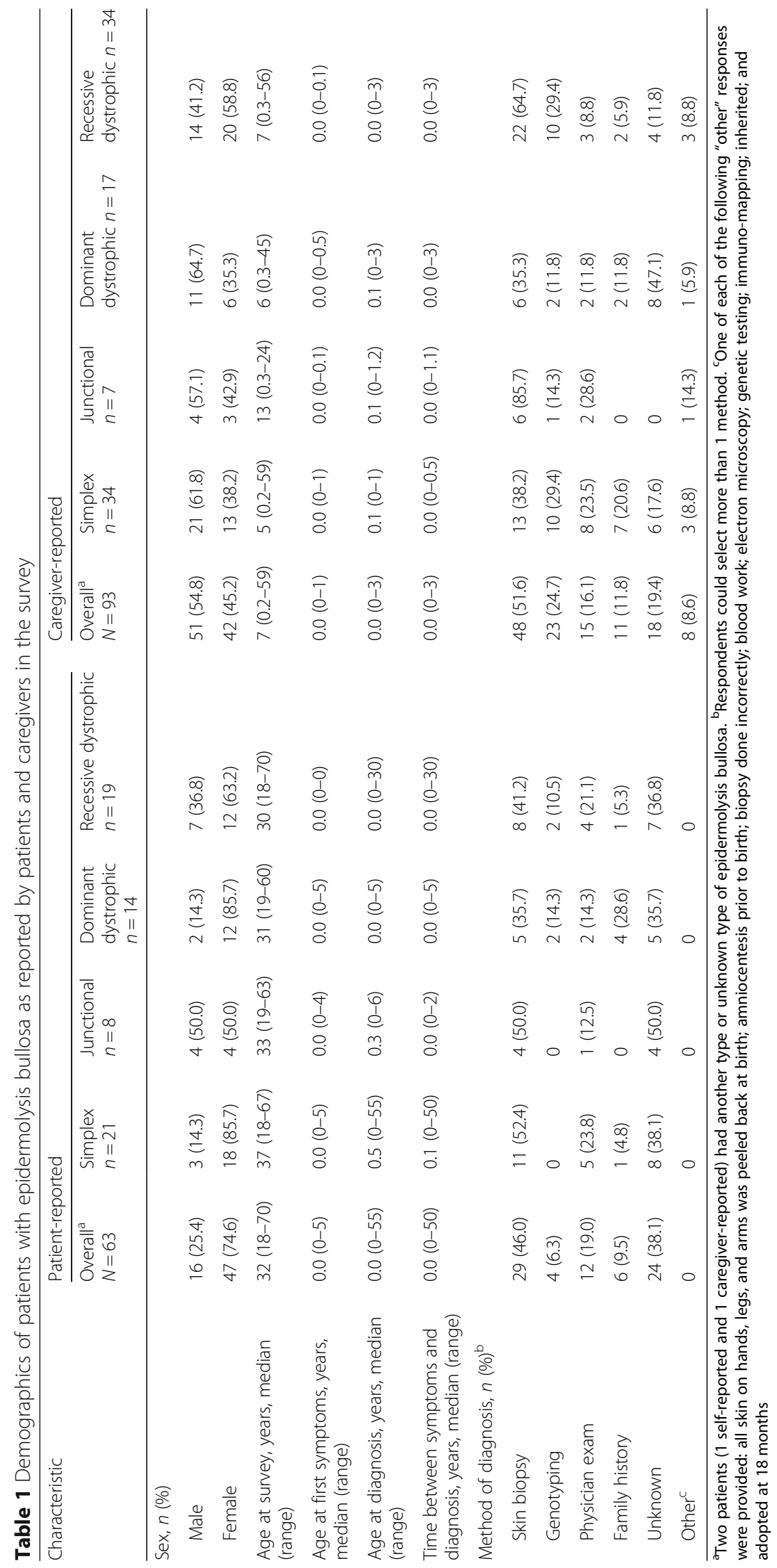




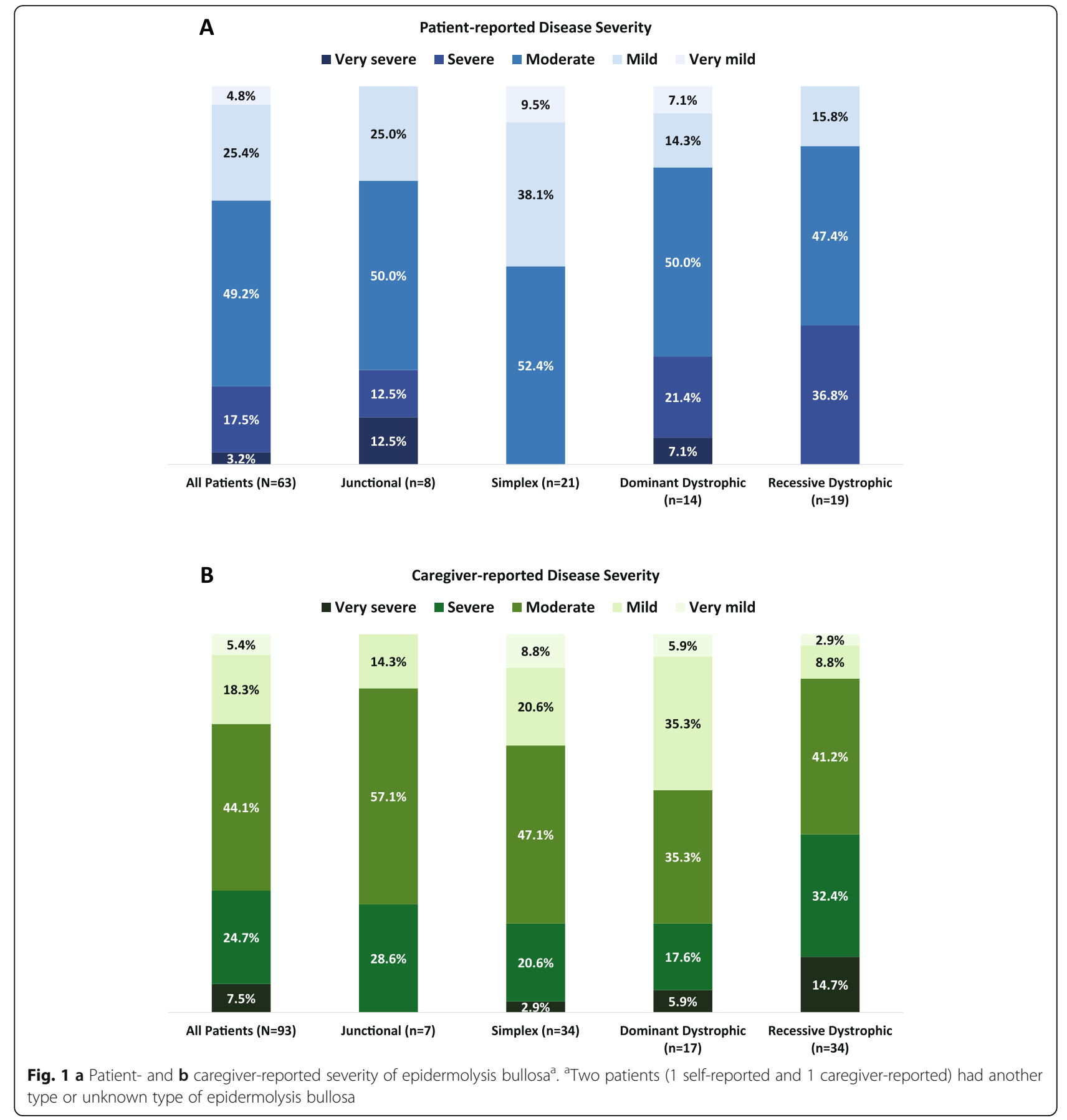

necessarily require assistance with wound care), physical disability, family preference, etc. According to patients, the most helpful sources of information for learning to care for wounds were a family member $(69.8 \%)$ or personal experience (trial and error; 39.7\%) (respondents could select $\geq 1$ source), whereas caregivers benefited almost equally from personal experience (32.3\%), patient community (30.1\%), family member (26.9\%), and epidermolysis bullosa specialists at epidermolysis bullosa treatment centers (25.8\%). Most respondents were extremely satisfied $(11.1 \%$ of patients $[n=7]$ and $17.2 \%$ of caregivers $[n=16])$, satisfied $(39.7 \%$ of patients $[n=25]$ and $23.7 \%$ of caregivers $[n=22]$ ), or somewhat satisfied (9.5\% of patients $[n=6]$ and $9.7 \%$ of caregivers $[n=9])$ with the wound care guidance received from healthcare specialists.

When respondents were asked to identify the most important factors for a future approved prescription treatment option, the top 5 responses among patients and caregivers were the same: reducing the risk of skin 


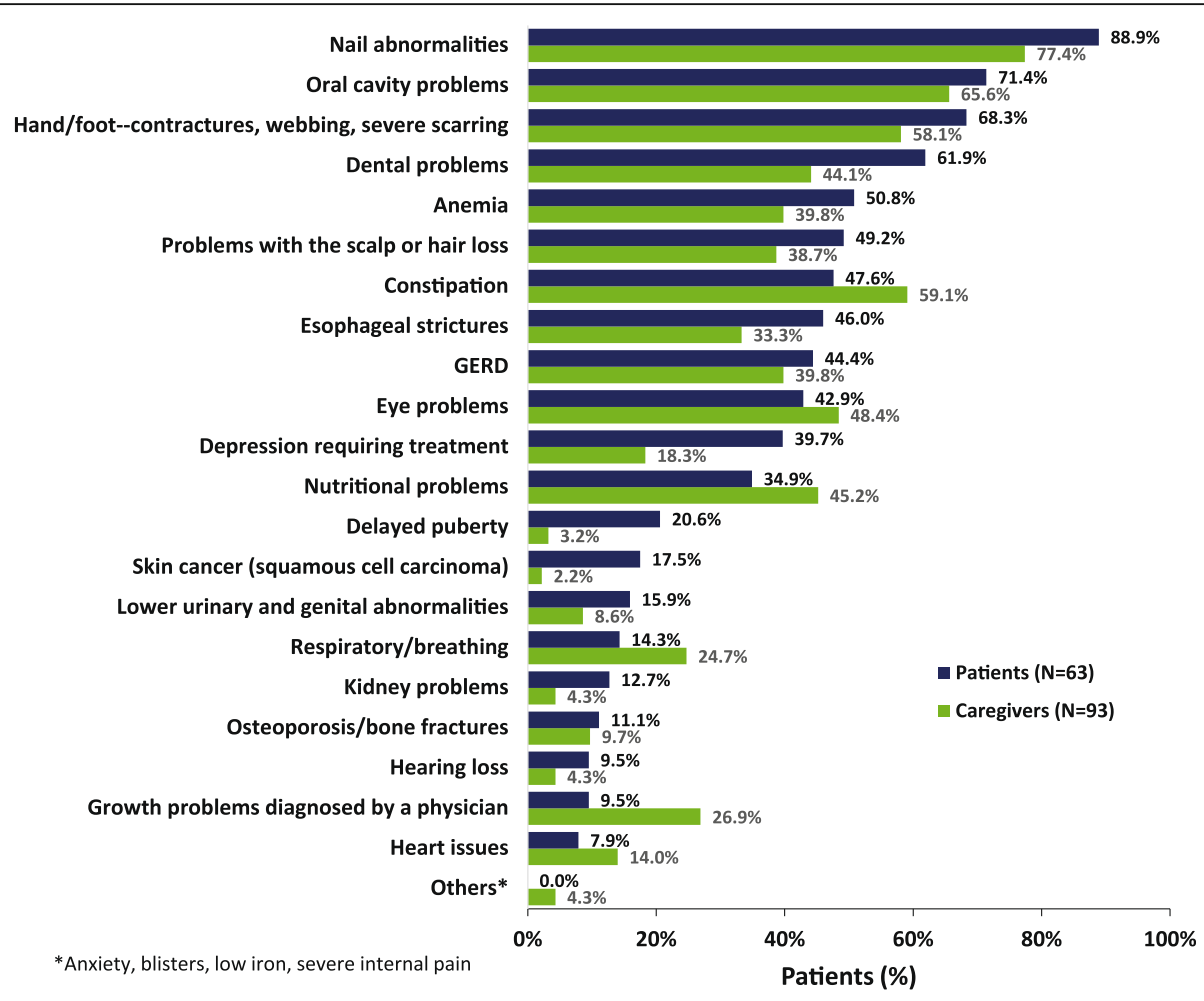

Fig. 2 Patient- and caregiver-reported complications experienced due to epidermolysis bullosa. Respondents ticked all applicable answers. aOthers: anxiety $(n=1)$; blisters $(n=1)$; low iron $(n=1)$; severe internal pains, cause unknown $(n=1)$. GERD gastroesophageal reflux disease

cancer $(77.8$ and $86.0 \%)$, reducing the number and severity of wounds (73.0 and $87.1 \%)$, reducing pain $(73.0$ and $78.5 \%)$, accelerating wound healing/closure (71.4 and $80.6 \%$ ), and reducing risk of infection (69.8 and $76.3 \%)$. Reducing itch (57.1 and 74.2\%) and decreasing time for dressing change (41.3 and $57.0 \%$ ) were more important for caregivers than for patients.

\section{Impact of epidermolysis bullosa on life decisions Patient-reported impact of epidermolysis bullosa on their life decisions}

The effects of epidermolysis bullosa can be far-reaching, with patients and caregivers making difficult life choices to deal with the impact of the disease. Of 63 patients who answered the question "What life decisions have you made based on epidermolysis bullosa?" all reported working fewer hours because of epidermolysis bullosa; $56(88.9 \%)$ reported that their disease influenced their career choice, and 46 (73.0\%) had decided not to work (Fig. 4a).

\section{Caregiver-reported impact of epidermolysis bullosa on their life decisions}

Of 93 caregivers who answered the question "If you are a parent of a child with epidermolysis bullosa, what life decisions have you made based on your child's epidermolysis bullosa?" most reported a profound impact on their life choices, with many deciding to reduce their working hours $(n=65 ; 69.9 \%)$ or give up work entirely ( $n=53 ; 57.0 \%$ ) (Fig. $4 \mathrm{~b})$.

Family planning was an important issue, to reduce the chances of having more children with epidermolysis bullosa. Many parents of children with epidermolysis bullosa reported that the disease was a factor in a divorce or separation ( $n=43,46.2 \%)$.

\section{Impact of epidermolysis bullosa on quality of life}

Both patients and caregivers reported that epidermolysis bullosa impacted the patient's quality of life and that a broad range of daily activities were negatively affected. Epidermolysis bullosa clearly interfered with patients' ability to move around their home, bathe or shower, write, eat, sleep, shop, participate in sports, and play (Table 4). Epidermolysis bullosa also caused physical pain beyond that stemming from wounds, with $39.7 \%$ of patients and $48.4 \%$ of caregivers reporting occasional pain, $22.2 \%$ of patients and $15.1 \%$ of caregivers reporting constant pain, and $15.9 \%$ of patients and $11.8 \%$ of caregivers reporting frequent pain, for themselves and for the patient in their care, respectively. To assess the emotional burden of epidermolysis bullosa on the patient, patients rated for themselves and caregivers rated for the 


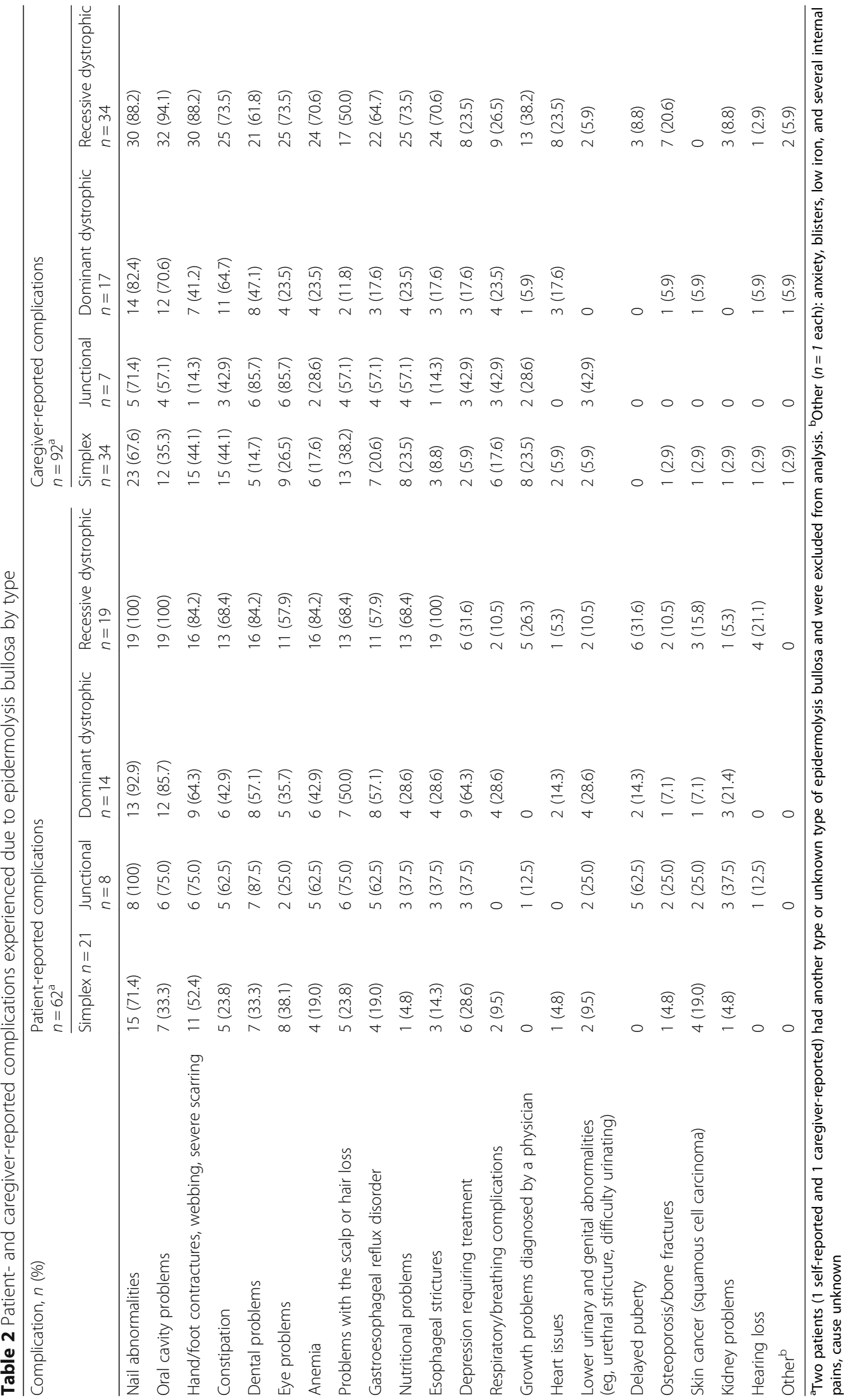




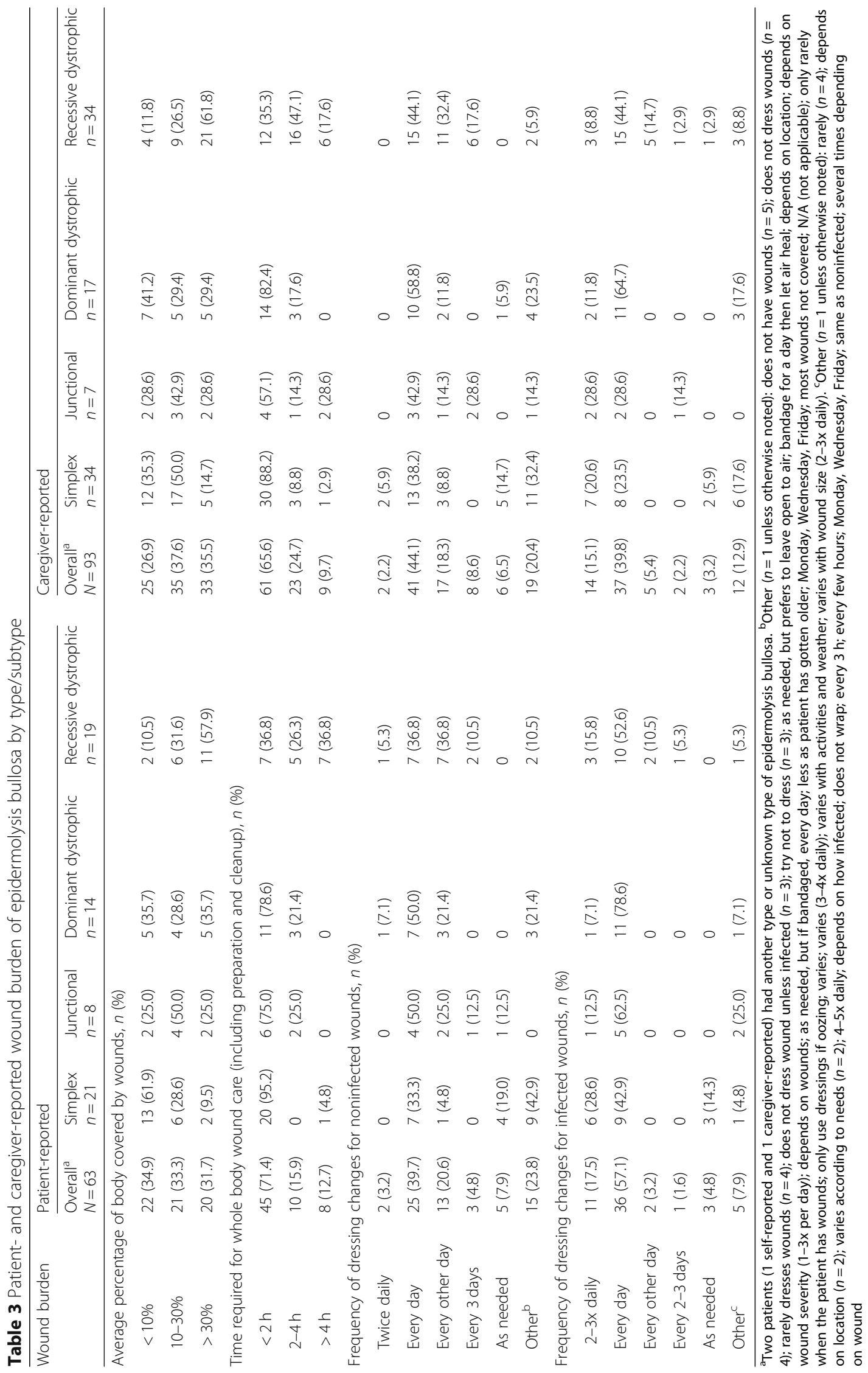



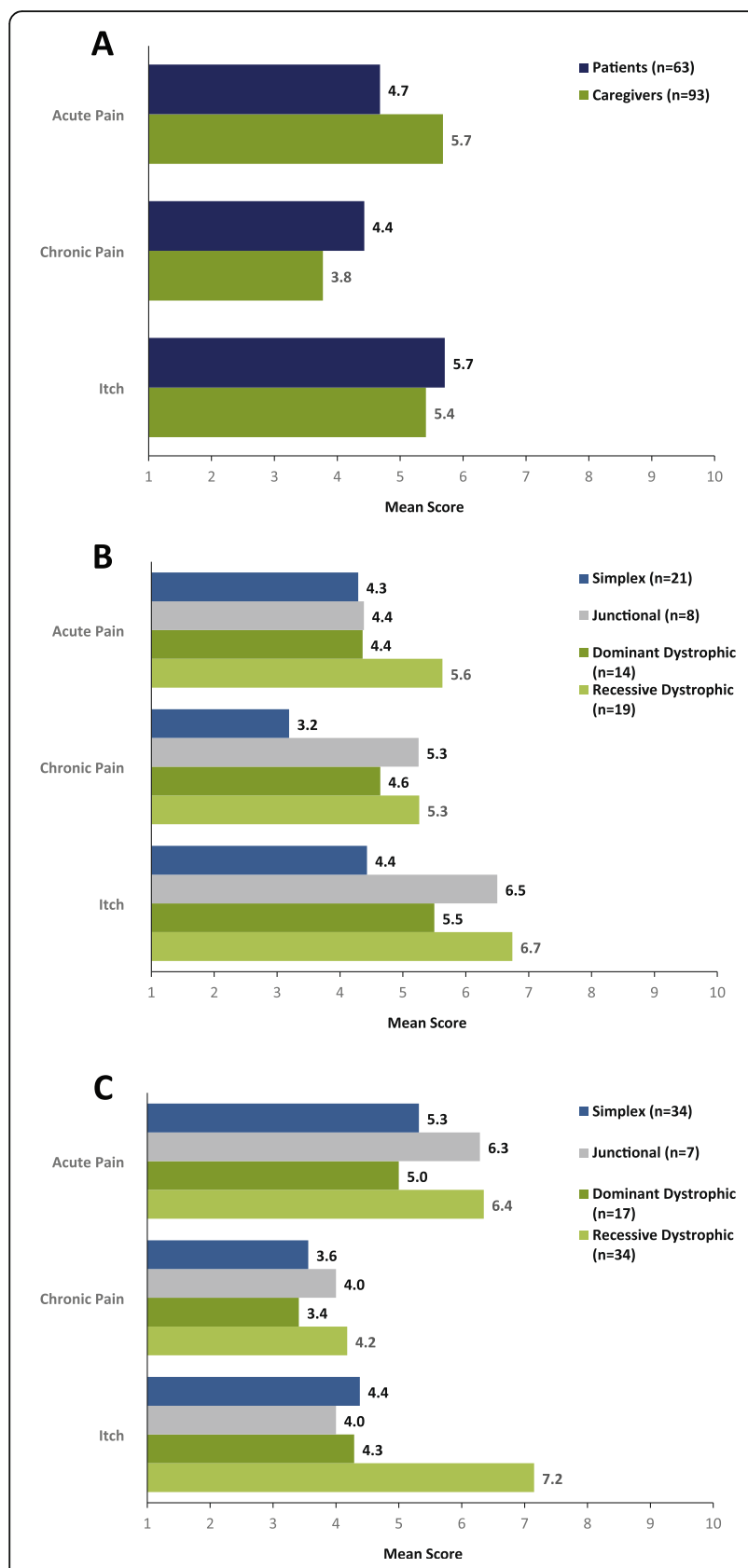

Fig. 3 Intensity of wound pain and itch (scored from 1 to 10) in the past 2 weeks as reported by a patients and caregivers, $\mathbf{b}$ as reported by patients by type, and $\mathbf{c}$ as reported by caregivers by type ${ }^{a}{ }^{a}{ }^{a} O n e$ patient had another type or unknown type of epidermolysis bullosa and was excluded from analysis

patient in their care, the level of frustration, embarrassment, worry/anxiety, and depression on a scale of 1 to 10 where 1 was defined as "do not feel" and 10 was defined as "feel very strongly." A higher level of negative emotions was reported by the patient themselves than what caregivers estimated for the patient. A rating of $\geq 5$ was reported by $88.9 \%$ of patients and $82.8 \%$ of caregivers for frustrated, $74.6 \%$ of patients and $52.7 \%$ of caregivers for embarrassed, $69.8 \%$ of patients and $57.0 \%$ of caregivers for worried or anxious, and $66.6 \%$ of patients and $34.4 \%$ of caregivers for depressed. Finally, epidermolysis bullosa negatively affected socialization of the patient, such as the ability to make new friends, with $44.4 \%$ of patients and $32.3 \%$ of caregivers reporting "a lot" of or "extensive" impact.

\section{Financial burden}

Survey results indicate that epidermolysis bullosa causes financial burden for a majority of patients and their caregivers. Thirty-two percent (31.7\%) of patients and $20.4 \%$ of caregivers reported "a lot" of financial burden from epidermolysis bullosa; in addition, $22.2 \%$ of patients and $37.6 \%$ of caregivers reported "a moderate amount." Most patients had health care coverage (95.2\% of patient respondents and $96.8 \%$ of patients whose caregivers responded). Among patient respondents, the most common types of health care coverage were commercial through employer (36.5\%) and Medicare (27.0\%) or Medicaid (23.8\%). Among caregivers, the most common types of health care coverage for the patient were commercial through employer (52.7\%), and Medicaid (41.9\%). However, not all epidermolysis bullosa-related expenses were reimbursed. The most commonly reported expenses that were not reimbursed by a healthcare plan were over-the-counter medications (81.0 and $77.4 \%$ as reported by patients and caregivers, respectively), dressing and wound supplies (65.1 and 63.4\%), prescription medications (44.0 and 38.7\%), and physician visits (38.1 and 32.3\%). The mean amount (USD) of unreimbursed expenses incurred for epidermolysis bullosa care was $\$ 262.34$ and $\$ 682.16$ per month as reported by patients and caregivers, respectively.

\section{Discussion}

Epidermolysis bullosa is a rare disease that causes significant morbidity and mortality to affected individuals $[1,4]$. Previous reports indicate that epidermolysis bullosa has a significant negative impact on health-related quality of life and places a substantial socioeconomic burden on patients with epidermolysis bullosa and their caregivers [12-16]. The survey reported herein aimed to obtain insight into the impact of epidermolysis bullosa on daily life and activities of patients and their caregivers, and to better understand the different types/subtypes of the condition.

The results of the survey indicate that epidermolysis bullosa places a considerable burden on patients. Many patients and caregivers (21 and 32\%) reported that the disease was severe or very severe, and the majority reported at least one additional complication. Furthermore, $19 \%$ of patients and $25.8 \%$ of caregivers had sought care for epidermolysis bullosa in an emergency 


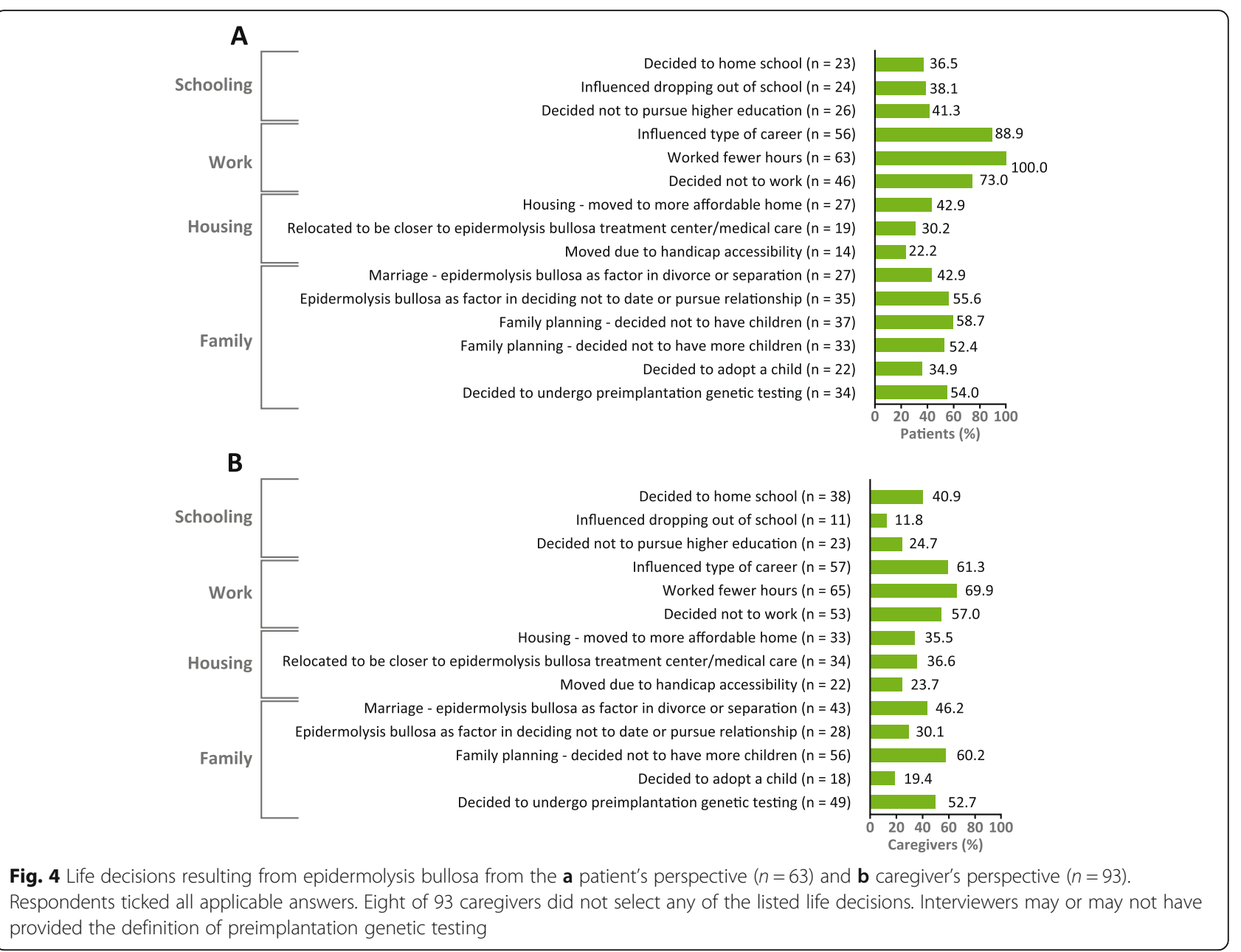

department in the 12 months prior to the survey. Some differences were seen between the different types/subtypes of the condition: patients with recessive dystrophic epidermolysis bullosa were more likely to report wounds on $>30 \%$ of their body surface and severe/very severe symptoms compared with patients with epidermolysis bullosa simplex $(57.9 \%$ vs $9.5 \%$ and $37 \%$ versus $0 \%$, respectively, based on patients' response). These findings are consistent with observations in the clinical setting, where recessive dystrophic epidermolysis bullosa is regarded as the most severe epidermolysis bullosa type beyond the neonatal/early infancy period.

Wound care was reported to be time consuming and commonly required caregiver assistance. Again, patients with recessive dystrophic epidermolysis bullosa reported the greatest disease burden, spending the longest amount of time on wound care ( $37 \%$ spent $>4 \mathrm{~h} /$ day), and recording the highest levels of acute pain (5.6 out of 10) and itch (6.7 out of 10). Most patients with epidermolysis bullosa and caregivers stated that they had learned to care for wounds by trial and error, or from family members and the patient community rather than from healthcare professionals, suggesting that this is an aspect of disease management that requires more attention at the time of initial diagnosis and during subsequent clinic visits. However, of those who did receive wound care guidance from specialists, most were satisfied with the outcome.

It is clear that both patients with epidermolysis bullosa and their caregivers must make difficult choices and compromises regarding education, career, and personal life. Often, the limitations caused by epidermolysis bullosa result in decreased academic and professional achievements, the consequences of which add to the burden of epidermolysis bullosa. Although this survey was not designed to evaluate the full range of psychological effects of the condition, patients with disfiguring skin conditions have reported suffering from poor selfesteem, anxiety, and depression; caregivers report feelings of stress, guilt, and isolation, further impacting overall well-being [4, 14]. Results of our survey support these observations, with patients and caregivers reporting that epidermolysis bullosa negatively impacts activities of daily living, socialization, and emotional well- 


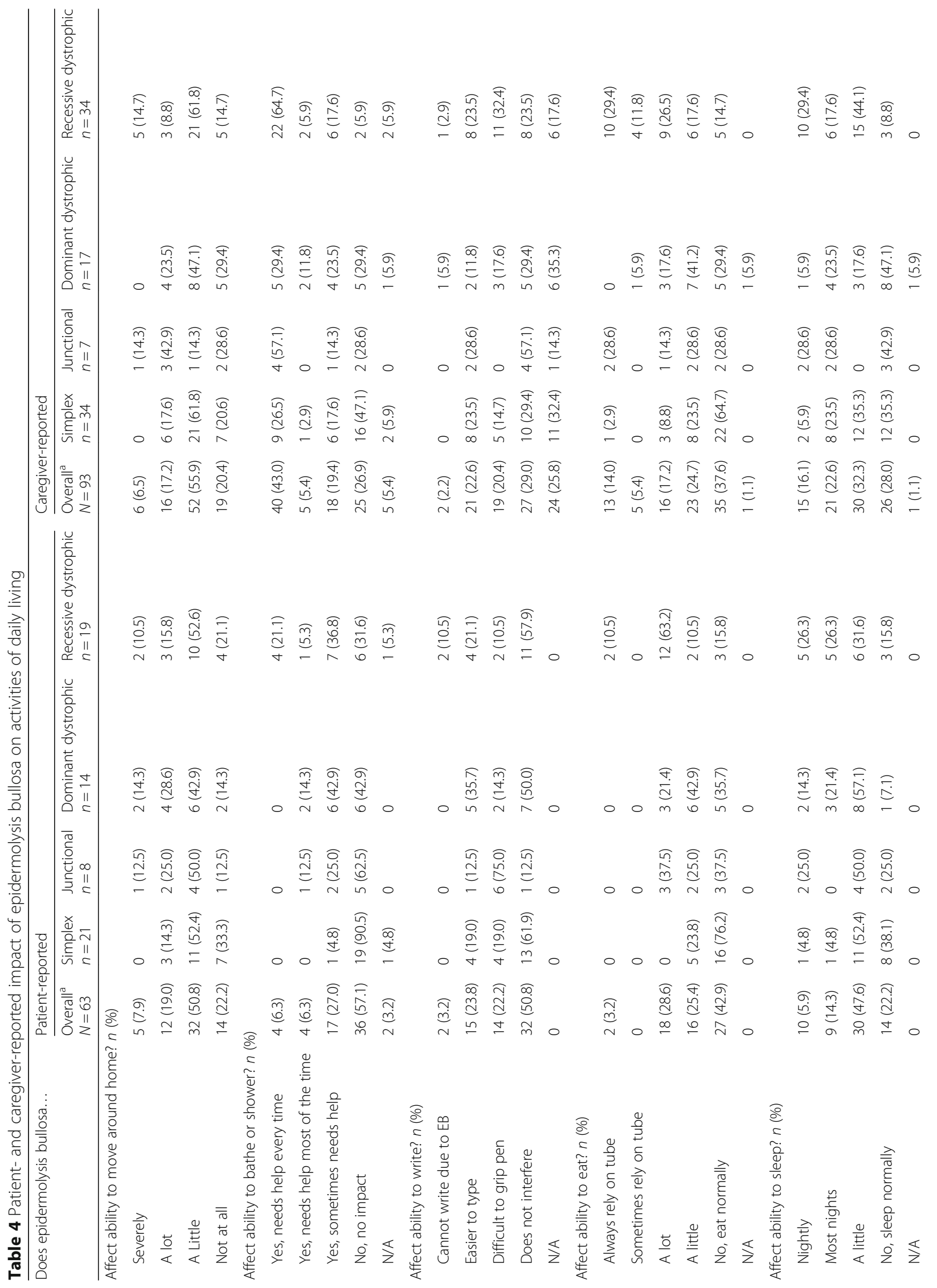




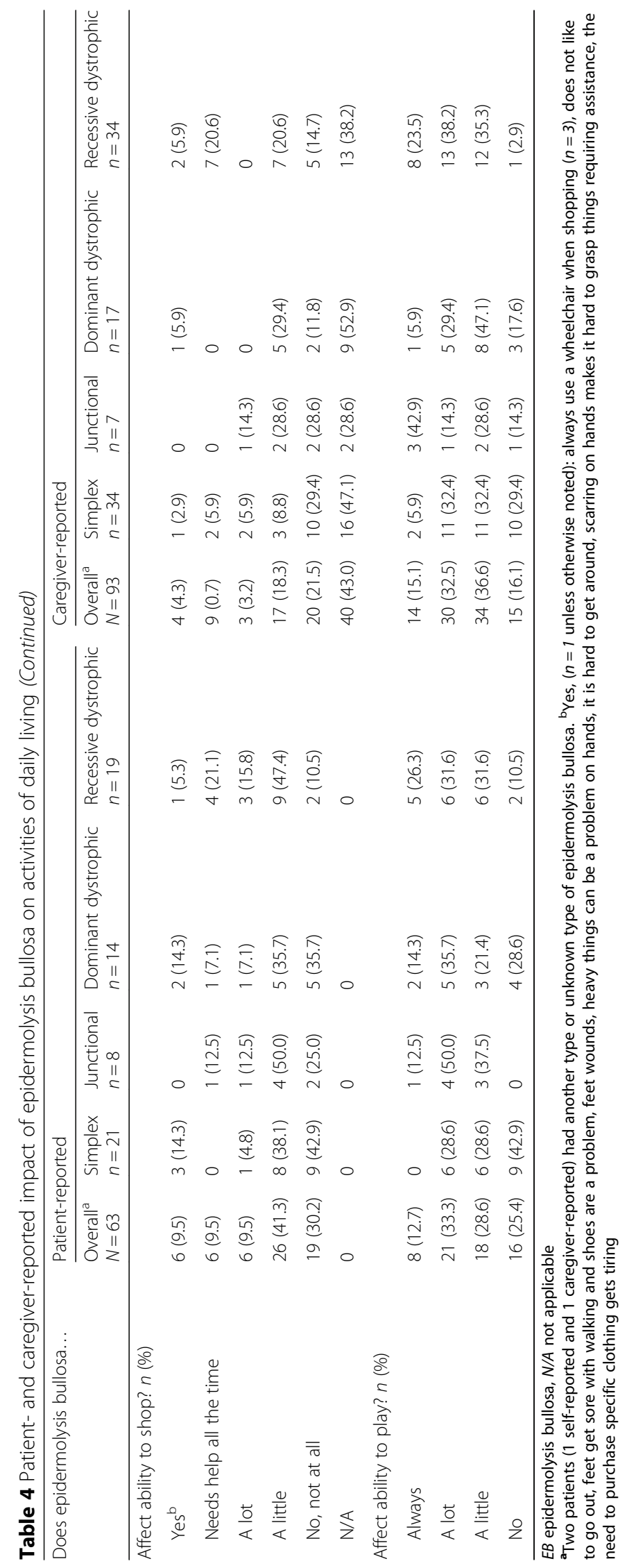


being. Finally, survey results suggest that the additional expenses incurred by epidermolysis bullosa cause a financial burden for patients, their caregivers, and their families. Our results are in agreement with those from a recent survey of 60 families affected by epidermolysis bullosa in France, which found that parents of children with epidermolysis bullosa experience substantial social, professional, and economic burden [17].

Despite advances in the understanding of the underlying pathophysiology of epidermolysis bullosa, to date, no treatments have been approved by regulatory authorities $[3,9]$. Current clinical trials for potential targeted therapies include gene therapy, cell-based therapies, and protein replacement therapy $[1,3]$. Patients and caregivers in this survey, and in prior publications [18], have indicated that reducing the number and severity of wounds and decreasing pain are among the main priorities in addition to reducing the risk of skin cancer, suggesting a potential key therapeutic role for topical creams or gels, several of which are also in clinical development [4].

Although surveys can be excellent tools to elicit feedback from patients and identify aspects of the care experience that may need improvement, they also have inherent limitations. One limitation of all surveys is low participation rate, which may suggest a selection bias. In the current study, more than a quarter of initial respondents who expressed interest in participation ultimately failed to complete the survey. Data bias due to question non-responses may also exist. In addition, the reliability of survey data is dependent on the accuracy of the answers provided. Responses provided by caregivers on behalf of patients may be different from those provided by patients themselves. Survey answers could lead to erroneous data because certain options (particularly subjective ratings) may be interpreted differently by respondents; in addition, respondents may not feel comfortable providing honest answers that present themselves in an unfavorable manner. No statistical tests were performed, limiting the comparison of different epidermolysis bullosa types/subtypes.

\section{Conclusions}

These survey results provide valuable information on the commonalities and differences for different types/subtypes of epidermolysis bullosa and confirm that the disease places a substantial burden on patients, their caregivers, and their families. Among the types/subtypes represented, patients and caregivers reported that recessive dystrophic epidermolysis bullosa had the greatest wound burden and acute pain, including the highest rating of disease severity and the highest percentage of body surface area covered by wounds. Wound care is time consuming and commonly requires caregiver assistance. Reducing the number and severity of wounds, pain, and risk of skin cancer were ranked among the most important factors in a future treatment option. The limitations caused by epidermolysis bullosa mean that both patients and caregivers must make difficult choices and compromises regarding schooling, work, housing, relationships, and family planning. Epidermolysis bullosa also negatively impacts quality of life and causes financial burden for patients, their caregivers, and their families.

\section{Supplementary information}

Supplementary information accompanies this paper at https://doi.org/10. 1186/s13023-019-1279-y

Additional file 1. Patient Survey. Copy of patient survey

Additional file 2. Patient Enrollment Map. US map showing number of enrolled patients per state.

\section{Abbreviations}

N/A: Not applicable; USD: United States dollars

\begin{abstract}
Acknowledgements
The authors thank the patients and caregivers who participated in the survey. The authors also acknowledge Engage Health for performing the survey and Metrics for Learning for analyzing the survey results. Third-party medical editorial assistance was provided by Sally-Anne Mitchell, PhD, of ApotheCom (Yardley, PA) and was supported by Amicus Therapeutics, Inc. (Cranbury, NJ)
\end{abstract}

\section{Author contributions}

ALB and DFM designed the patient survey; reviewed/analyzed the data; critically revised the paper. ML drafted survey questions; oversaw and managed survey development and review process; facilitated IRB approval; oversaw and participated in data analysis; and critically revised the manuscript. JW contributed to the study design, survey development, data analysis and critically revised the manuscript. NP contributed to the survey design and data analysis. AR assisted in designing the survey, contributed to the analysis of the data, and critically reviewed the paper. JGa critically revised the paper. BK critically revised the paper and participated in patient recruitment. $\mathrm{MH}$ performed the analyses. $\mathrm{HL}$ reviewed and revised draft survey questions and approved the final questionnaire; conceived and designed the analyses; reviewed the results of the analysis and drafted conclusions; critically revised the paper. JGe contributed to writing of the paper; critically revised the paper, contributed to developing and reviewing the survey questions. All authors read and approved the final manuscript.

\section{Funding}

Funding for the survey was provided by Amicus Therapeutics, Inc. The survey design, analyses, interpretation of the data and writing of the manuscript were done in collaboration with the authors. The survey was executed by Engage Health.

\section{Availability of data and materials}

The datasets used and/or analyzed during the current study are available from the corresponding author on reasonable request.

\section{Ethics approval and consent to participate}

The survey was approved by a central institutional review board (New England Independent Review Board). All patients or caregivers provided informed consent to participate in the survey.

Consent for publication

Not applicable. 


\section{Competing interests}

ALB has served as a consultant and an investigator for Amicus Therapeutics/ Scioderm and Castle Creek, and as an investigator for Amryt, Fibrocell, ProQR, and Phoenix Tissue Repair.

JW, NP, AR, HL, ML, and JGa, are employees of and hold stock in Amicus Therapeutics.

JGe is the co-founder and vice chair on the board of Profession Patient Advocates in Life Sciences; a member of the board of trustees for the Healthcare Institute of New Jersey; a member of the board of directors for the Together Strong Foundation for NPC, and an employee of, and a stock holder and patent holder in, Amicus Therapeutics.

BK is an employee of Debra of America.

$\mathrm{MH}$ is an employee of EB Research Partnership.

DFM has served as an investigator for Amicus Therapeutics, Amryt, Castle

Creek, and Shire, and has served as an advisor for Amicus and Shire.

\section{Author details}

'Children's Hospital Colorado, University of Colorado School of Medicine, 13123 E 16th Ave, B570, Aurora, CO 80045, USA. ${ }^{2}$ Amicus Therapeutics, Inc, 1 Cedar Brook Drive, Cranbury, NJ 08512, USA. ${ }^{3}$ Debra of America, 75 Broad St \#300, New York, NY 10004, USA. ${ }^{4}$ EB Research Partnership, 132 East 43rd St, Suite 432, New York, NY 10017, USA. ${ }^{5}$ St. George-Hospital, University of Sydney, NSW 2052, Australia.

Received: 16 July 2019 Accepted: 16 December 2019

Published online: 03 January 2020

\section{References}

1. El Hachem M, Zambruno G, Bourdon-Lanoy E, Ciasulli A, Buisson C, HadjRabia S, et al. Multicentre consensus recommendations for skin care in inherited epidermolysis bullosa. Orphanet J Rare Dis. 2014;9:76.

2. Fine JD. Inherited epidermolysis bullosa. Orphanet J Rare Dis. 2010;5:12.

3. Ma JE, Hand JL. What's new with common genetic skin disorders? Minerva Pediatr. 2017;69:288-97.

4. Tabor A, Pergolizzi JV Jr, Marti G, Harmon J, Cohen B, Lequang JA. Raising awareness among healthcare providers about epidermolysis bullosa and advancing toward a cure. J Clin Aesthet Dermatol. 2017:10:36-48.

5. Fine JD, Bruckner-Tuderman L, Eady RA, Bauer EA, Bauer JW, Has C, et al. Inherited epidermolysis bullosa: updated recommendations on diagnosis and classification. J Am Acad Dermatol. 2014;70:1103-26.

6. Debra International. Epidermolysis bullosa 2018. http://www.debrainternational.org/homepage.html. Accessed 9 Apr 2019.

7. Fine JD. Epidemiology of inherited epidermolysis bullosa based on incidence and prevalence estimates from the National Epidermolysis Bullosa Registry. JAMA Dermatol. 2016;152:1231-8.

8. Bruckner-Tuderman L, JA MG, Robinson EC, Uitto J. Progress in epidermolysis bullosa research: summary of DEBRA International research conference 2012. J Invest Dermatol. 2013;133:2121-6.

9. Uitto J, Bruckner-Tuderman L, Christiano AM, JA MG, Has C, South AP, et al. Progress toward treatment and cure of epidermolysis bullosa: summary of the DEBRA International research symposium EB2015. J Invest Dermatol. 2016:136:352-8.

10. Goldschneider KR, Good J, Harrop E, Liossi C, Lynch-Jordan A, Martinez AE, et al. Pain care for patients with epidermolysis bullosa: best care practice guidelines. BMC Med. 2014;12:178.

11. von der Lippe C, Diesen PS, Feragen KB. Living with a rare disorder: a systematic review of the qualitative literature. Mol Genet Genomic Med. 2017;5:758-73.

12. Angelis A, Kanavos P, Lopez-Bastida J, Linertova R, Oliva-Moreno J, SerranoAguilar $\mathrm{P}$, et al. Social/economic costs and health-related quality of life in patients with epidermolysis bullosa in Europe. Eur J Health Econ. 2016; 17(suppl 1):31-42.

13. Pagliarello C, Tabolli S. Factors affecting quality of life in epidermolysis bullosa. Expert Rev Pharmacoecon Outcomes Res. 2010;10:329-38.

14. Jain SV, Murrell DF. Psychosocial impact of inherited and autoimmune blistering diseases. Int J Womens Dermatol. 2018;4:49-53.

15. Tabolli S, Pagliarello C, Uras C, Di Pietro C, Zambruno G, Castiglia D, et al. Family burden in epidermolysis bullosa is high independent of disease type/subtype. Acta Derm Venereol. 2010;90:607-11.

16. Frew JW, Martin LK, Nijsten T, Murrell DF. Quality of life evaluation in epidermolysis bullosa (EB) through the development of the QOLEB questionnaire: an EB-specific quality of life instrument. $\mathrm{Br} J$ Dermatol. 2009; 161:1323-30.

17. Dufresne H, Hadj-Rabia S, Bodemer C. Impact of a rare chronic genodermatosis on family daily life: the example of epidermolysis bullosa. Br J Dermatol. 2018;179:1177-8.

18. Davila-Seijo P, Hernandez-Martin A, Morcillo-Makow E, de Lucas R, Dominguez $\mathrm{E}$, Romero $\mathrm{N}$, et al. Prioritization of therapy uncertainties in dystrophic Epidermolysis Bullosa: where should research direct to? An example of priority setting partnership in very rare disorders. Orphanet $J$ Rare Dis. 2013;8:61.

\section{Publisher's Note}

Springer Nature remains neutral with regard to jurisdictional claims in published maps and institutional affiliations.

\section{Ready to submit your research? Choose BMC and benefit from:}

- fast, convenient online submission

- thorough peer review by experienced researchers in your field

- rapid publication on acceptance

- support for research data, including large and complex data types

- gold Open Access which fosters wider collaboration and increased citations

- maximum visibility for your research: over $100 \mathrm{M}$ website views per year

At BMC, research is always in progress.

Learn more biomedcentral.com/submissions 